\title{
Conservative management of intracranial extradural haematoma presenting late
}

\author{
R ILLINGWORTH, ${ }^{*}$ H SHAWDON† \\ From the Regional Department of Neurosurgery* and Department of Radiology, $\uparrow$ Central Middlesex \\ Hospital, London, UK
}

SUMMARY Three patients with intracranial extradural haematomas presenting several days after injury have been managed without surgery, with clinical improvement. Spontaneous resolution of the haematomas has been demonstrated by serial computed tomography over periods of six weeks.

Intracranial extradural haematoma is usually fatal unless diagnosed and treated promptly. The diagnosis is made in most patients within 24 hours of injury ${ }^{1}$ but presentation may be delayed until the second or third week. ${ }^{2}$ In one series $93 \%$ of patients presented within the first seven days, ${ }^{1}$ and in another $84 \%$ presented within the first six days, ${ }^{2}$ but $7 \%$ of children and $4 \%$ of adults presented in the third week. The operative mortality is higher in patients presenting in the first 24 hours, being $30 \%$ in the series of McKissock et al. $^{2}$ and $15 \%$ in the later series of Jamieson and Yelland. ${ }^{1}$ In contrast only 1 of 33 patients presenting late died. ${ }^{3}$ Operation is believed to be required for all cases and has been advised in asymptomatic patients presenting late. ${ }^{4}$

Computed tomography (CT) is the safest and most reliable method of demonstrating intracranial haematomas, and by serial scanning over a period of time, changes in size and density may be observed. This may allow a patient with an extradural haematoma who is showing clinical improvement to be managed without surgery if progressive resolution of the haematoma can be shown. A previous paper $^{5}$ has described the CT appearances and clinical course of two patients with small temporal extradural haematomas managed conservatively. We present three further patients, two of whom had temporal haematomas and one a large frontal haematoma, managed without operation.

Address for reprint requests: Mr RD Illingworth, Central Middlesex Hospital, Acton Lane, Park Royal, London, NW10 7NS, UK

Received 18 December 1982

Accepted 26 January 1983

\section{Case reports}

Case 1 A 40-year-old man received a head injury in a road traffic accident in Nigeria. There was transient loss of consciousness with post-traumatic amnesia of about two days and a shorter period of retrograde amnesia. A laceration in the right parietal region was sutured. He was discharged from hospital after a few days but continued to have severe headaches, worse in the mornings. When first seen two weeks after the injury, he was still complaining of headache, although this was improving. He was fully conscious and no abnormal neurological signs could be found. Skull radiographs showed a fracture in the right parietal region and CT 15 days after injury showed an extradural haematoma underlying the fracture (fig A). In view of the improvement in his headache it was decided not to operate. Two weeks later his headache had completely cleared and CT 42 days after injury showed almost complete resolution of the haematoma (fig B).

Case 2 A seven-year-old child received a head injury when she fell off a wall. There was probably only transient loss of consciousness and after being seen in the casualty department of her local hospital she was sent home. She remained drowsy and irritable and then developed diplopia, which led to her being brought back to the hospital. Skull radiographs showed a fracture and she was transferred to this Department of Neurosurgery. On admission, four days after injury, she was a little drowsy and irritable and reluctant to answer questions. There were mild bilateral VIth nerve palsies, but no other abnormal signs. The following day her conscious level had improved and she was no longer irritable. CT showed a left frontal extradural haematoma (fig C). In view of the experience with Case 1 it was decided to attempt conservative management. Within two days her mental state and alertness had returned to normal, though diplopia persisted for several more days. A repeat scan after one week showed the haematoma to be 


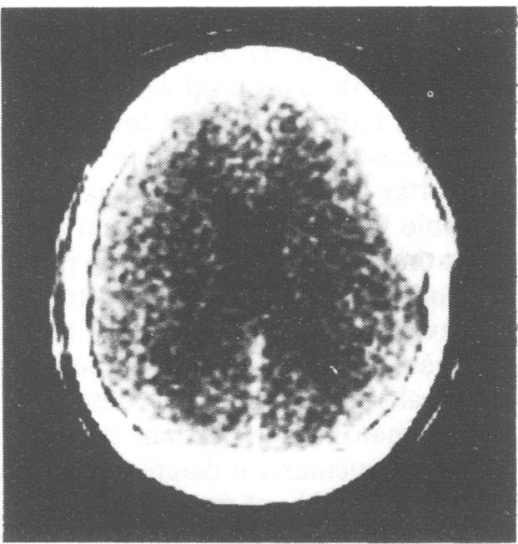

Fig A Case 1: CT 15 days after injury

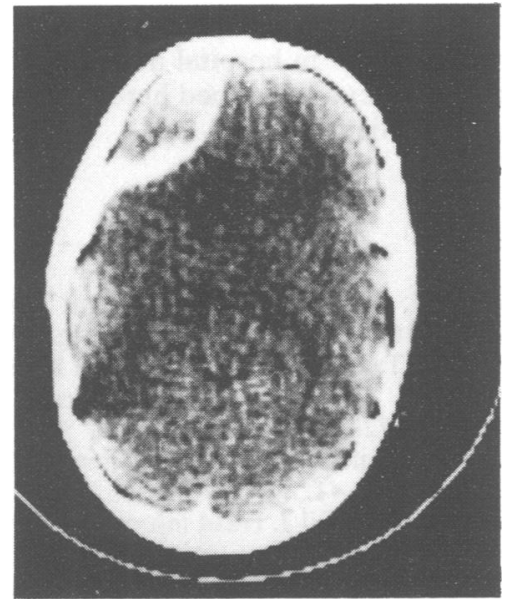

Fig C Case 2: CT 5 days after injury

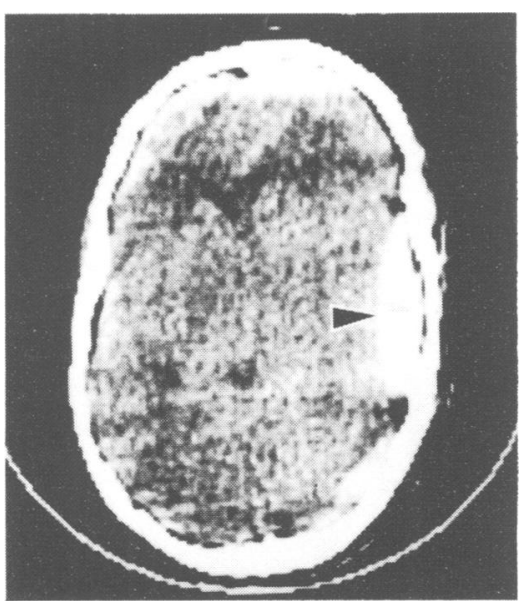

Fig E Case 3: CT on the day of injury

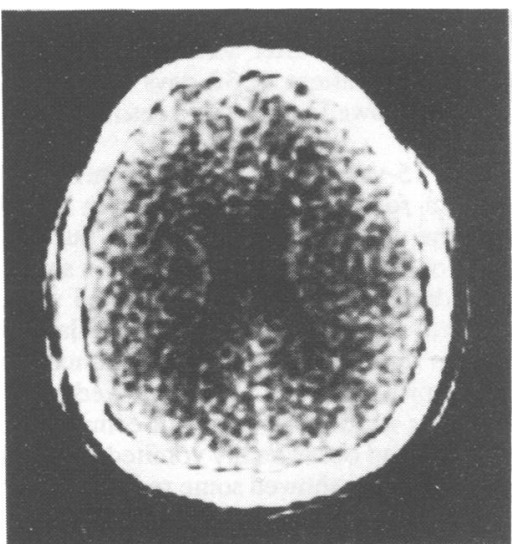

Fig B Case 1: CT 42 days after injury

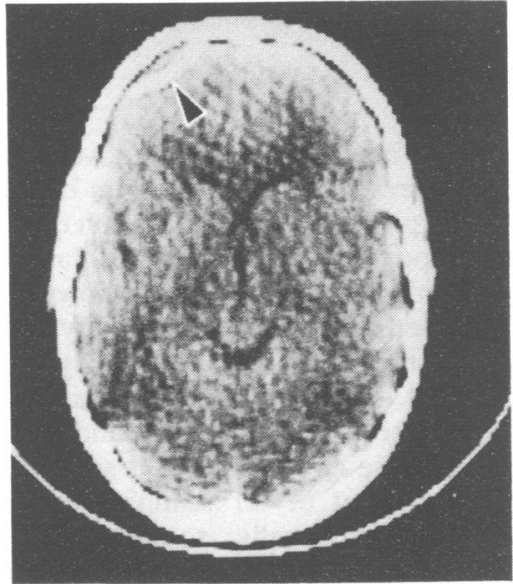

Fig D Case 2: CT 52 days after injury

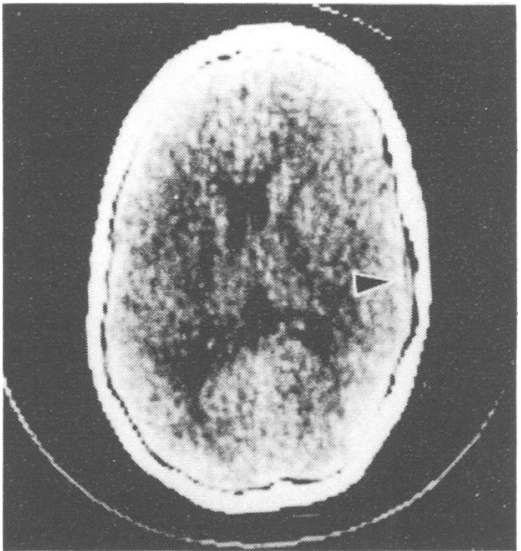

Fig F Case 3: CT 46 days after injury 
the same size, but much less dense. She was discharged from hospital 14 days after admission. Further scans performed as an outpatient at 32 days and at 52 days after injury (fig D) showed progressive resolution.

Case 3 A 15-year-old girl was admitted after being involved in a road accident while riding her bicycle. On admission she was drowsy and restless, but obeying commands. CT on the day of injury showed a small right temporal extradural haematoma and marked swelling of the right cerebral hemisphere with ventricular compression and midline shift (fig E). The appearances suggested a fairly severe primary injury. She improved rapidly and was fully conscious within two days, but complained of persistent headache and occasionally vomited. A repeat CT scan ten days after injury showed some reduction in the cerebral swelling, but slight increase in the size of the extradural haematoma. In view of the clinical improvement it was felt that evacuation of the haematoma was not indicated. She was discharged home 12 days after admission, and rescanned to show resolution of the haematoma after 46 days (fig F).

\section{Discussion}

Although the majority of patients with extradural haematoma present within 24 hours of injury and usually show progressive deterioration of consciousness, a proportion presents much later. Urgent operation is mandatory in patients showing progressive clinical deterioration and morbidity and mortality are, to a large extent, related to delay in diagnosis and treatment. However, the prognosis for patients presenting later is much better, and the surgical mortality in these circumstances is small.

Until the advent of CT scanning an extradural haematoma was usually sought when clinical deterioration occurred, by surgical exploration without resource to major neuroradiological investigation which, except in doubtful cases, may only waste time and delay surgery. CT allows intracranial haematomas to be rapidly and accurately localised and operation can then proceed with confidence that the correct area has been chosen for surgery. However, with increased access to CT for the screening of patients after injury, more haematomas which appear suitable for surgical evacuation may be discovered, but the clinical progress of the patients may not always indicate the need for such treatment.

We believe that although the majority of extradural haematomas will continue to require urgent surgical evacuation, the detection of an extradural haematoma in a patient showing clinical improvement, particularly if diagnosed at some time after the injury, should not necessarily be an indication for evacuation. It may be possible to manage such patients conservatively in anticipation of absorption of the haematoma, which in the patients described here occurred over a period of up to six weeks. CT can monitor the progress of this, but the period of time spent in hospital need only be short, and all three patients described here were managed mainly as outpatients.

\section{References}

1 Jamieson KG, Yelland JDN. Extradural haematoma: Report of 167 cases. J Neurosurg 1968;299:13-23.

${ }^{2}$ McKissock W, Taylor JC, Bloom WH, Till K. Extradural haematoma: Observation of 125 cases. Lancet 1960;2:167-72.

${ }^{3}$ Young TW. Chronic extradural haematoma. J Clin Practice 1972;26:38-41.

${ }^{4}$ Polo JM, Ibanez A, Gil J. Head injury and CT scanning. Lancet 1980;1:720.

${ }^{5}$ Weaver D, Pobereskin L, Jane JA. Spontaneous resolution of epidural haematomas. Report of two cases. $J$ Neurosurg 1981;54:248-51. 